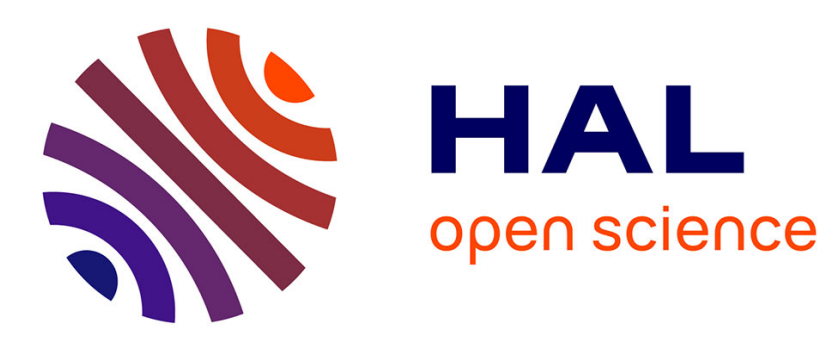

\title{
Carrières et relations de pouvoir chez les médecins hospitaliers : une comparaison européenne,
} Carine Vassy

\section{To cite this version:}

Carine Vassy. Carrières et relations de pouvoir chez les médecins hospitaliers: une comparaison européenne,. Geneviève Cresson et François-Xavier Schweyer (dir.) Professions et institutions de santé face à l'organisation du travail. Aspects sociologiques., Editions de l'Ecole Nationale de Santé Publique, p.71-86, 2000. hal-01278842

\section{HAL Id: hal-01278842 \\ https://hal.science/hal-01278842}

Submitted on 24 Feb 2016

HAL is a multi-disciplinary open access archive for the deposit and dissemination of scientific research documents, whether they are published or not. The documents may come from teaching and research institutions in France or abroad, or from public or private research centers.
L'archive ouverte pluridisciplinaire HAL, est destinée au dépôt et à la diffusion de documents scientifiques de niveau recherche, publiés ou non, émanant des établissements d'enseignement et de recherche français ou étrangers, des laboratoires publics ou privés. 
Carine Vassy (2000) Carrières et relations de pouvoir chez les médecins hospitaliers : une comparaison européenne, in G. Cresson et F.X. Schweyer (dir.) Professions et institutions de santé face à l'organisation du travail. Aspects sociologiques. Rennes : Editions de l'Ecole Nationale de Santé Publique, p.71-86

Les travaux d'Oswald Hall (1970) ont montré l'importance des rapports de pouvoir entre les chefs de services et les autres médecins hospitaliers dans les années 40 aux Etats-Unis. Les seconds dépendent largement des premiers pour faire carrière dans les hôpitaux, comme pour réussir leur installation en médecine libérale. Il s'agit d'un système de patronage, fondé sur la compétition des médecins à l'intérieur de chaque discipline, pour accéder à certains postes clés. Progresser dans la carrière nécessite d'avoir un patron dans le «noyau central » de la profession (Hall, 1970). C'est cette élite médicale qui contrôle l'entrée de nouveaux candidats, qui en évince certains, qui en récompense d'autres, faisant respecter des règles qui modèlent le visage de la profession. Le patronage est aussi un échange de services, le patron aidant ses protégés à franchir les différentes étapes de la carrière médicale, et ceux-ci acceptant des charges de travail et des périodes probatoires importantes dans leurs différents postes hospitaliers. Le système de patronage existe-t-il avec les mêmes caractéristiques en Europe ? La répartition des postes dans le champ hospitalier obéit-elle à des mécanismes identiques d'un pays à l'autre ?

Dans une vaste enquête comparative sur les hôpitaux dans le monde, William Glaser (1963) a suggéré que le pouvoir des patrons hospitaliers sur leurs subordonnés était plus ou moins important selon les pays. Il fait l'hypothèse que dans les pays européens la dépendance des médecins juniors est plus importante qu'aux Etats-Unis, du fait de l'étroitesse des marchés du travail nationaux, et du cumul des chefs de service européens, qui ont des responsabilités à la fois administrative, clinique, pédagogique et scientifique. Des travaux de sociologie des organisations ont été menées par la suite sans mettre en évidence de différences nationales. Olgierd Kuty (1973) et Marianne Binst (1988) montrent que les stratégies médicales en milieu hospitalier ne présentent pas de différence notable entre la Belgique et la France d'une part, les Etats-Unis, la Suisse et la France d'autre part.

A la suite de ces travaux, nous avons étudié les relations de travail dans six services de neurologie en Allemagne (Munich et Francfort), France (Paris et banlieue parisienne) et Grande-Bretagne (Londres et Cambridge) de 1990 à 1993 (Vassy, 1997). Les six services de soins font partie d'hôpitaux universitaires. Les missions attribuées au personnel sont identiques : soigner des patients, former le personnel stagiaire ou temporaire, et améliorer les connaissances médicales au sein de la discipline. L'enquête sociologique a consisté à interviewer l'ensemble du personnel médical et soignant, en centrant les entretiens sur l'organisation du service, et à observer différentes situations de travail. Dans certains cas, les résultats de cette recherche ont été exposés aux personnes interviewées et ont suscité un débat.

Pour simplifier la présentation des médecins hospitaliers rencontrés dans les trois pays, nous avons défini des catégories d'analyse spécifiques qui désignent à la fois les étapes qu'ils ont franchies dans leur formation, leur rôle dans l'organisation du travail, leur place dans la hiérarchie hospitalière et leur avancement dans la carrière professionnelle. A l'aide de ces critères, on peut distinguer trois catégories : la haute hiérarchie médicale du service, les médecins seniors et les médecins juniors.

Le sommet de la hiérarchie médicale est constitué en France et en Allemagne du chef de service (Chefarzt), et en Grande-Bretagne des consultants (Consultants). En Allemagne et en France, une 
seule personne détient ces responsabilités, tandis qu'en Grande-Bretagne, la direction du service est assurée par plusieurs consultants qui se partagent la responsabilité juridique de la prise en charge des patients hospitalisés dans le service. Dans les trois pays, ce sont des médecins spécialistes de neurologie, titulaires de leur poste, responsables juridiquement du service, et parvenus au sommet de la hiérarchie médicale hospitalière. Ils ont une activité clinique, une activité d'enseignement dans et hors le service, et pour certains une activité de recherche. Ils ont un grand nombre d'activités extérieures au service : consultations, participation à des commissions au sein de l'hôpital, de l'université ou de l'administration de la santé, participation à des colloques, éventuellement enseignement à l'université de médecine et recherche etc. Ils délèguent la responsabilité du suivi quotidien des patients à des médecins subordonnés, tout en contrôlant les principales décisions cliniques lors des visites des patients qu'ils effectuent toutes les semaines ou tous les quinze jours.

Nous avons regroupé dans la catégorie des médecins seniors les médecins chefs en Allemagne (Oberarzt), en France les chefs de clinique et les Praticiens Hospitaliers (PH) encore appelé "adjoint" (du chef de service), et en Grande-Bretagne les médecins certifiés (Registrar) et les médecins certifiés seniors (Senior Registrar). Ce sont des médecins diplômés, spécialistes de neurologie, recrutés par le sommet de la hiérarchie médicale du service pour avoir à la fois des responsabilités cliniques et d'enseignement, et éventuellement pour faire de la recherche. Ils ne sont pas parvenus au sommet de la hiérarchie hospitalo-universitaire, mais cette perspective est encore ouverte pour la plupart d'entre eux ${ }^{1}$, et ils sont sous la responsabilité juridique de la haute hiérarchie médicale. Ils sont responsables d'une partie des patients du service (en général 10 à 20 patients). Ils ont eux aussi de nombreuses activités extérieures au service, comme l'enseignement, les consultations etc. Ils délèguent le suivi des patients aux médecins juniors, qu'ils supervisent en passant quotidiennement dans le service et en faisant avec eux le tour des patients une ou plusieurs fois par semaine.

Enfin nous avons regroupé dans la catégorie des médecins juniors les médecins stagiaires (Arzt Im Praktikum) et les médecins assistants (Assistenz Arzt) ${ }^{2}$ en Allemagne, les internes de spécialité et les internes de médecine générale en France, et les médecins résidents (Senior Health Officer) en Grande-Bretagne. Ce sont les médecins du service les plus récemment diplômés. Ils ont obtenu leur diplôme de médecin à l'université, mais n'ont pas encore le droit d'exercer la médecine sans supervision. Leur travail clinique est contrôlé par les autres médecins. Ils ne peuvent prescrire que pour les patients du service et n'ont pas d'ordonnancier à leur nom. Dans le service, ce sont les médecins les plus présents. Ils sont chargés du suivi journalier des patients : admission, examens cliniques, prescriptions d'examens, intervention immédiate en cas d'urgence etc.

L'analyse des relations de travail entre les différents médecins a permis de montrer qu'il existait trois types de services. Le premier type rassemble les services de Cambridge et de la banlieue parisienne. Les médecins sont satisfaits de l'activité du service. La haute hiérarchie médicale a acquis une réputation internationale dans sa discipline du fait de ses travaux de recherche sur une pathologie neurologique. Du fait de sa spécialisation, elle accueille des patients présentant des cas complexes, envoyés par d'autres neurologues répartis sur l'ensemble du territoire national. Elle a recruté comme médecins seniors des neurologues qui ont déjà travaillé dans des services réputés et

\footnotetext{
${ }^{1}$ Les médecins seniors peuvent tous progresser dans la carrière hospitalo-universitaire, sauf le praticien hospitalier qui ne peut accéder à une chefferie de service. Comme lui, les médecins-chefs allemands peuvent rester à leur poste jusqu'à l'âge de la retraite car ils bénéficient de contrat de travail à durée indéterminée. Mais ils peuvent aussi passer l'habilitation et briguer un poste de chef de service. Les chefs de clinique français et les médecins certifiés britanniques n'ont qu'un contrat de travail d'une durée de quelques années, mais peuvent encore progresser dans la carrière.

${ }^{2}$ La différence entre le médecin stagiaire et le médecin assistant est leur ancienneté. Après dix-huit mois de travail dans le service, le médecin stagiaire peut être recruté comme médecin assistant.
} 
qu'elle trouve de bon niveau. Les médecins seniors apprécient l'activité clinique spécialisée et la plupart d'entre eux font des travaux de recherche. Cette activité, ainsi que l'appui de leur patron, leur ouvre des perspectives de carrières hospitalières. Les médecins juniors, pour leur part, se félicitent de la formation clinique qu'ils reçoivent de leurs collègues, ainsi que de la possibilité qui leur est offerte d'étudier des cas pathologiques rares. Ils ont des chances sérieuses d'être recrutés sur des postes de médecins seniors dans leur service, ou dans d'autres services d'hôpitaux universitaires.

Dans le deuxième type de services, à Francfort et à Paris, les relations de travail sont beaucoup moins aisées. Les médecins seniors reprochent aux chefs de service, proches de l'âge de la retraite, de ne pas avoir d'activité de recherche et de ne pas leur donner les moyens d'en faire. Leurs perspectives de carrière sont de ce fait limitées. Comme le service n'a pas de réseau de correspondants neurologues, les patients sont le plus souvent envoyés par les médecins généralistes des environs de l'hôpital. Les médecins soignent le «tout-venant» des maladies neurologiques, ce qui les prive d'occasions d'étude et d'apprentissage. Les médecins juniors critiquent aussi leur formation sous un autre angle : ils trouvent que le chef de service les encadre peu. Une partie d'entre eux ne veut pas se spécialiser dans cette discipline et les autres s'inquiètent de leur faible probabilité de carrière hospitalière.

Le troisième type de services rassemble ceux de Munich et Londres et présente des caractéristiques intermédiaires. La haute hiérarchie médicale effectue des activités de recherche et a des responsabilités scientifiques internationales. Mais sa politique de spécialisation est contrée par plusieurs facteurs. A Munich, les neurologues peinent à hospitaliser des cas rares parce qu'ils subissent la concurrence de leurs collègues du second hôpital universitaire de la ville et celle des nombreux neurologues libéraux qui ont développé la prise en charge des patients en secteur ambulatoire. Les neurologues libéraux, fortement représentés dans les caisses d'assurance maladie, ont également bloqué les tarifs de remboursement des consultations hospitalières, ce qui freine le développement des consultations externes de l'hôpital. A Londres, le service dirigé par les neurologues n'est pas considéré dans l'hôpital comme un service spécialisé. Il est soumis à l'obligation d'hospitaliser des patients relevant d'autres disciplines, en particulier ceux provenant du service des urgences. Les neurologues ne peuvent pas spécialiser totalement le recrutement des patients et n'ont pas assez d'alliés parmi leurs confrères dans l'établissement pour obtenir le titre de service spécialisé.

Dans ces deux services, la haute hiérarchie médicale incite les médecins seniors à faire des travaux de recherche pendant qu'ils sont embauchés par l'hôpital ou en dehors de ces périodes, en leur fournissant des contrats de recherche. Les médecins seniors ont déjà une certaine reconnaissance scientifique et des perspectives de carrière en milieu hospitalo-universitaire. Les médecins juniors en revanche sont moins satisfaits de leur sort : ils estiment accomplir une charge de travail considérable auprès des patients, du fait de l'absence des médecins seniors qui effectuent une multitude d'activités autres que cliniques. Ils jugent être insuffisamment payés en retour car la haute hiérarchie médicale ne recrute qu'une petite partie d'entre eux pour les postes de médecins seniors, et elle n'hésite pas à embaucher des candidats venant d'autres hôpitaux universitaires.

Cette typologie montre que les relations de travail dans le groupe médical ne présentent pas de caractéristiques nationales. Les trois types rassemblent des services indépendamment de leur nationalité. Il s'agit donc d'expliquer à la fois les points communs observés, qui sont à l'origine de l'absence de spécificités nationales, et les différences qui séparent les trois types.

\section{Pourquoi l'absence de spécificités nationales?}


La qualité des relations de travail dans le groupe des médecins hospitaliers dépend des possibilités de progression dans la carrière. Les relations qui s'établissent dans ce groupe sont fortement liées aux perspectives de formation, d'activités et de revenus qui s'ouvrent aux uns et aux autres. Ces différentes facettes de la carrière médicale sont régies par des règles qui varient peu dans les trois pays européens considérés. Nous allons en analyser quelques unes.

\section{Une sélection progressive}

L'organigramme du groupe médical se présente comme une pyramide dans les services allemands et français, et comme un marteau dans les services britanniques, où le nombre de consultants est supérieur au nombre de médecins juniors et seniors. Mais quelle que soit la forme de l'organigramme, dans tous les services étudiés, le nombre de candidats à chaque passage d'un poste à l'autre est supérieur au nombre de postes à pourvoir.

Dans les trois pays, la démographie médicale est telle qu'il y a beaucoup plus de médecins en formation que de postes hospitaliers à pourvoir. Les services étudiés se caractérisent par un très grand nombre de candidatures, du simple fait d'appartenir à des établissements hospitalouniversitaires. Aux candidats internes, ayant déjà travaillé dans le service, s'ajoutent des candidats externes. Le déséquilibre entre l'offre d'emplois et la demande s'accentue encore du fait que les niveaux supérieurs de la hiérarchie restent beaucoup plus longtemps à leur poste que les niveaux subordonnés. La durée du séjour dans le service se compte en semaine ou en mois pour les étudiants, en semestre ou en année pour les médecins juniors et certains seniors, et sans limite temporelle pour certains médecins seniors et le sommet de la hiérarchie médicale, qui sont titulaires de postes fixes.

A chaque franchissement d'un niveau hiérarchique, une sélection des candidats est opérée soit par le sommet de la hiérarchie médicale du service, soit par des examens universitaires, soit enfin par une commission comprenant des médecins travaillant ou non dans le service, voire des non médecins. Il peut y avoir aussi une combinaison des ces différents modes de sélection. Nous allons voir que le pouvoir de décision du sommet de la hiérarchie du service est prépondérant dans la plupart des cas, et que les candidats retenus sont souvent des candidats internes.

\section{Le pouvoir de décision du sommet de la hiérarchie médicale}

Les médecins parvenus au sommet de la hiérarchie hospitalo-universitaire ont la responsabilité de recruter les médecins seniors qui travaillent dans le service, ainsi que les médecins juniors en Allemagne et en Grande-Bretagne, et même les étudiants en stage dans l'un des services allemands. La haute hiérarchie médicale du service détient donc la clé de la progression professionnelle des autres médecins, qu'elle sélectionne par étapes successives.

Dans notre échantillon, la haute hiérarchie médicale dit privilégier le critère des travaux de recherche effectués par les candidats, mais aussi prendre en compte les compétences cliniques et les qualités relationnelles. La qualité des travaux est évaluée par le nombre de publications et la réputation des médias utilisés, comme dans d'autres professions intellectuelles (Caplow, Mc Gee ; 1968) ${ }^{3}$.

\footnotetext{
3 On peut comparer la situation des enseignants universitaires et celle des médecins hospitalo-universitaires, car les uns et les autres sont évalués sur leurs travaux de recherche, alors qu'une grande partie de leur temps est consacrée à une autre activité, enseigner dans un cas, soigner et former dans l'autre.
} 


\section{La valorisation de la carrière hospitalo-universitaire}

Ce processus de sélection a comme condition nécessaire la motivation des médecins du bas de la hiérarchie à faire une carrière hospitalière. La grande majorité des médecins juniors interviewés veut continuer à exercer une activité spécialisée à l'hôpital, activité ressentie comme l'une des plus attrayantes du fait de son intérêt intellectuel. La motivation pour la carrière hospitalière va de pair avec une forte valorisation de l'activité de recherche. Celle-ci est présentée comme une activité intellectuelle stimulante permettant de participer à la création du savoir médical, ou comme une possibilité d'agir pour le bien des malades futurs, alors qu'on ne peut sauver les malades présents, ou encore comme une échappatoire au tête-à-tête angoissant avec le patient. La préférence pour les postes hospitaliers n'empêche d'ailleurs pas une attitude critique par rapport au fonctionnement de l'hôpital et aux règles de la communauté médicale. La pratique libérale a la réputation d'offrir des avantages en terme d'indépendance et de rémunération, mais de ne pas donner la possibilité de rester à la pointe du progrès médical.

Le désir de poursuivre une carrière hospitalière s'accompagne d'une hiérarchie de la valeur des postes et des modes d'exercice qui est semblable chez les médecins juniors interviewés dans les trois pays. Les postes les plus recherchés sont ceux des hôpitaux universitaires, si possible dans les services qui ont une activité de recherche importante, puis les postes dans les hôpitaux qui n'ont pas d'activité de recherche, puis l'exercice de la médecine libérale. Nous retrouvons là une observation de Michel Arliaud à propos des médecins français. L'auteur qualifie d'hospitalo-centrisme cette "hiérarchie matérielle et symbolique des positions professionnelles qui caractérise la médecine contemporaine et dont le centre hospitalo-universitaire constitue le sommet" (Arliaud, 1987, p.90). Les services des hôpitaux universitaires, qui sont les plus recherchés, sont aussi les plus sélectifs.

\section{Le caractère local du recrutement}

Le marché du travail des neurologues hospitaliers est étroit. Mais cela ne suffit pas à expliquer que la plupart des carrières hospitalo-universitaires s'effectuent au sein de quelques services hospitaliers, voire d'un seul service. Le plus souvent, ce sont des candidats internes qui sont embauchés sur les postes de médecins seniors, voire sur les postes du sommet de la hiérarchie. La haute hiérarchie médicale préfère recruter les candidats qu'elle a déjà vu travailler dans son service. Cette pratique peut être rapprochée d'un mode d'embauche fréquent en entreprise, où l'on propose aux jeunes recrues des contrats à durée déterminée, avant de les recruter éventuellement avec des contrats à durée indéterminée. Les responsables testent ainsi l'adéquation du candidat au poste.

Le caractère endogène du recrutement augmente le pouvoir de la hiérarchie sur ses subordonnés. Ceux-ci travaillent au quotidien avec des personnes susceptibles de les recruter ultérieurement ou de les recommander pour d'autres postes. Même s'ils quittent le service, ils ne peuvent pas se permettre d'être en de mauvais terme avec leur hiérarchie car ils ont besoin d'être recommandés par elle ${ }^{4}$. La probabilité d'être embauché dans un service dans lequel le candidat n'a jamais travaillé est toutefois faible car il est en concurrence avec les candidats internes. De plus ses possibilités de mobilité dépendent de la réputation des médecins du service qu'il quitte. Si leur réputation est mauvaise, il n'a aucune chance de retrouver un poste dans un autre service hospitalo-universitaire.

\footnotetext{
${ }^{4}$ Quand il y a un recrutement de candidats externes, le sommet de la hiérarchie recrute sur dossier, à partir d'un certain nombre de pièces comprenant les résultats obtenus lors des études de médecine et des examens, les attestations de stages déjà effectués, éventuellement les travaux de recherche déjà accomplis, et les recommandations dont bénéficie le candidat. Les lettres de recommandation écrites sont très utilisées pour le recrutement des médecins juniors et seniors dans les deux services britanniques et pour l'embauche des médecins juniors dans les deux services allemands.
} 
Cet ensemble de facteurs fait que le ou les premiers services dans lesquels un médecin junior commence à travailler sont déterminants pour la suite de sa carrière, comme l'a relevé $\mathrm{O}$. Hall (1970) aux Etats-Unis. Les candidats aux postes de médecins juniors postulent en conséquence à des postes souvent éloignés de leur ville et de leur université d'origine.

Le tableau suivant montre que même s'il y a des variations dans les modes de recrutement selon les pays et les postes, le recrutement est souvent à la fois endogène et du ressort de la haute hiérarchie médicale. Dans certains cas, plusieurs instances sont consultées lors de l'embauche sans que cela atténue ces deux caractéristiques. Le recrutement des chefs de service dans les deux unités françaises en donne un exemple frappant ${ }^{5}$.

Tableau des modes de recrutement des médecins selon les services

\begin{tabular}{|l|l|l|l|}
\hline & $\begin{array}{l}\text { Allemagne : } \\
\text { services de Munich et } \\
\text { Francfort }\end{array}$ & $\begin{array}{l}\text { France : } \\
\text { services de Paris et Banlieue } \\
\text { parisienne }\end{array}$ & $\begin{array}{l}\text { Royaume-Uni : } \\
\text { services de Londres et Cambridge }\end{array}$ \\
\hline $\begin{array}{l}\text { Recrutement } \\
\text { des médecins } \\
\text { juniors }\end{array}$ & $\begin{array}{l}\text { Choix sur dossier, par le } \\
\text { chef de service, } \\
\text { éventuellement parmi } \\
\text { les anciens étudiants du } \\
\text { service. }\end{array}$ & Concours de l'internat & $\begin{array}{l}\text { Choix sur dossier, par les } \\
\text { consultants du service } \\
\text { et des consultants d'autres } \\
\text { services de l'hôpital. Le candidat } \\
\text { est recruté pour faire plusieurs } \\
\text { stages dans l'hôpital. }\end{array}$ \\
\hline $\begin{array}{l}\text { Recrutement } \\
\text { des médecins } \\
\text { seniors }\end{array}$ & $\begin{array}{l}\text { Choix parmi les anciens } \\
\text { médecins juniors du } \\
\text { service, par le chef de } \\
\text { service. }\end{array}$ & $\begin{array}{l}\text { Choix parmi les anciens } \\
\text { médecins juniors du service, } \\
\text { par le chef de service. }\end{array}$ & $\begin{array}{l}\text { Choix parmi les anciens médecins } \\
\text { juniors du service, ou des } \\
\text { candidats externes, par les } \\
\text { consultants réunis en commission. }\end{array}$ \\
\hline $\begin{array}{l}\text { Recrutement } \\
\text { du sommet de } \\
\text { la hiérarchie } \\
\text { médicale }\end{array}$ & $\begin{array}{l}\text { Choix sur dossier parmi } \\
\text { des candidats externes, par } \\
\text { une commission } \\
\text { comprenant une majorité } \\
\text { de professeurs de } \\
\text { l'université de médecine à } \\
\text { laquelle l'hôpital est } \\
\text { rattaché (Interdiction } \\
\text { juridique de recruter un } \\
\text { ancien médecin senior du } \\
\text { service). }\end{array}$ & $\begin{array}{l}\text { Choix parmi les anciens } \\
\text { médecins seniors du service, } \\
\text { par le chef de service qui va } \\
\text { quitter son poste, sous } \\
\text { réserve de l'approbation de } \\
\text { la CCM de l'hôpital, du } \\
\text { CNU, de la CME de l'AP- } \\
\text { HP et du Ministère de la } \\
\text { Santé. }\end{array}$ & $\begin{array}{l}\text { Choix sur dossier, parmi lens médecins seniors du } \\
\text { anciens } \\
\text { service ou des candidats externes, } \\
\text { par une commission comprenant } \\
\text { quelques consultants du service, } \\
\text { des représentants de l'hôpital et de } \\
\text { l'administration de la Santé. }\end{array}$ \\
\hline
\end{tabular}

En Grande-Bretagne, la proportion de recrutement endogène n'est qu'en apparence plus faible. Il est vrai que les médecins juniors étant recrutés pour faire trois à quatre stages semestriels dans le même hôpital, les consultants de neurologie ne sont pas seuls à prendre la décision de les recruter. Ils partagent cette prérogative avec d'autres consultants de l'établissement. De plus le recrutement des médecins seniors et du sommet de la hiérarchie peut s'effectuer parmi des candidats externes. Mais le recrutement est relativement endogène à cause du faible nombre des neurologues britanniques (170 consultants en 1990) et de l'organisation de la profession : les consultants travaillent souvent dans plusieurs hôpitaux. Un grand nombre d'entre eux travaillent à temps partiel à l'hôpital national de Queen Square, célèbre institut de neurologie londonien, où ils donnent des cours, assurent des

\footnotetext{
${ }^{5}$ Dans les deux services français, le chef de service actuel a été choisi par son prédécesseur, qui l'a fait nommer Professeur des Universités - Praticien Hospitalier quand il était encore en activité, puis qui l'a fait nommer à son poste au moment de son départ en retraite. La nomination a été entérinée par la Commission Consultative Médicale (CCM), organe de représentation des médecins dans l'hôpital, le Conseil National des Universités (CNU), et la Commission Médicale d'Etablissement (CME) de l'Assistance Publique, organe de représentation des médecins des différents hôpitaux de l'Assistance Publique de Paris. M. Binst et F. X. Schweyer (1995) ont analysé de manière critique les conséquences de la nomination des chefs de service par leur prédécesseurs dans différents hôpitaux français.
} 
consultations ou suivent leurs patients qui y sont hospitalisés. Ils sont amenés à connaître d'autres consultants, médecins seniors ou médecins juniors, qui y travaillent. Ils peuvent recruter comme médecin senior, ou même consultant, un médecin qui n'a pas travaillé dans leur service, mais dont ils connaissent les compétences directement, via le travail effectué dans cet institut, ou indirectement par la réputation qu'ils ont acquise auprès d'autres consultants de Queen Square. La présence dans la commission de recrutement de médecins qui n'appartiennent pas au service, et de non médecins, n'est pas déterminante. Dans les deux services britanniques, les derniers consultants recrutés étaient les candidats que les consultants en place souhaitaient embaucher.

Le pouvoir de la haute hiérarchie médicale sur les recrutements des autres médecins est prépondérant, hormis dans les deux cas exceptionnels que sont le recrutement du sommet de la hiérarchie médicale en Allemagne, et celui des médecins juniors en France. En Allemagne, une loi interdit de recruter comme chef de service un médecin qui a déjà travaillé en tant que médecin senior dans ce service. En France, le recrutement des internes s'effectue uniquement par concours. C'est le seul cas de tout notre échantillon où le recrutement est déterminé par les seuls résultats à des épreuves intellectuelles. Les internes, une fois qu'ils ont réussi le concours, choisissent euxmêmes les services dans lesquels ils vont faire leurs huit stages semestriels. Le chef de service ne les choisit pas, il est choisi par eux. Les internes exploitent les avantages que leur offre ce mode de recrutement : ils font leurs stages dans un grand nombre de services différents ${ }^{6}$ pour multiplier les occasions de se faire apprécier des chefs de service, et ils organisent l'échange mutuel d'informations sur les services 7 . Mais leur position de force reste toute relative, car l'accès aux postes de chefs de clinique, de praticien hospitalier et de praticien hospitalo-universitaire, se fait uniquement sur choix du chef de service.

\section{L'éviction vers la médecine libérale}

Dans les trois pays, les médecins évincés de la carrière hospitalière ont comme principal débouché la médecine libérale. Les conditions de leur exercice libéral dépendent de la manière dont ils ont été évalués par la haute hiérarchie médicale hospitalière. Plus ils sont évincés précocement du cursus hospitalier, moins les conditions d'exercice en tant que médecin libéral leur sont favorables.

En Allemagne et en France, où le secteur libéral comprend à la fois des médecins généralistes et des médecins spécialistes, l'observation montre que plus le médecin en formation est évincé du système de sélection hospitalier tardivement, plus il peut exercer des activités libérales spécialisées et rémunératrices. En Allemagne par exemple, l'installation comme médecin spécialiste est réservée aux anciens médecins chefs et médecins assistants ayant travaillé dans des services hospitaliers

\footnotetext{
${ }^{6}$ Cela est d'autant plus facile en région parisienne qu'il y a une douzaine d'hôpitaux universitaires disposant de services de neurologie.

7 Etant donné leur mode de recrutement, les internes ont des moyens de pression sur la haute hiérarchie médicale plus forts que les médecins juniors allemands et britanniques. Dans les trois pays, les médecins juniors mécontents de la politique de la hiérarchie médicale peuvent la sanctionner par une action collective décalée dans le temps. Ils peuvent faire une mauvaise réputation aux médecins du service auprès des autres médecins et des étudiants. Il s'ensuit une forme de "vote avec les pieds", les médecins juniors qui se sont montrés les plus brillants lors de leurs études ne postulant pas dans ces services. En France, les internes exploitent davantage ce moyen de pression du fait du mode d'attribution des stages. Ils organisent la diffusion des informations par l'intermédiaire du Syndicat des Internes qui tient un fichier constitué des rapports rédigés par ses membres sur leurs stages hospitaliers. Ce fichier est consultable uniquement par les internes. Comme ils choisissent chaque semestre leurs stages en donnant la priorité à ceux d'entre eux qui ont le plus d'ancienneté et le meilleur rang au concours, l'ancienneté des internes faisant un stage dans un service est un indicateur de la "côte du service", c'est-à-dire de sa réputation par rapport aux autres services de la même spécialité. Cette évaluation officieuse est généralement connue de tous les médecins de la spécialité.
} 
spécialisés et ayant réussi le concours de spécialisation. Les autres ne peuvent exercer que comme médecin généraliste. En France, la même logique est poussée à l'extrême. Si la personne qui a terminé ses études de médecine échoue au concours de l'internat, ou si elle renonce à passer ce concours, elle ne peut exercer qu'en tant que médecin généraliste. L'interne qui n'a pas obtenu de poste de chef de clinique, ou qui renonce à poursuivre sa formation hospitalière, peut s'installer en secteur libéral, mais il n'a pas l'accès automatique au secteur à honoraires libres, à la différence des anciens chefs de clinique. Dans ces deux pays, même le médecin qui veut exercer en secteur libéral est incité à gravir le plus possible les échelons de la carrière hospitalière.

En Grande-Bretagne, le processus est identique mais il prend une forme différente car le secteur libéral comprend très peu de médecins spécialistes. Seuls les consultants hospitaliers peuvent exercer à temps partiel une activité privée spécialisée. Celle-ci est donc réservée aux médecins parvenus au sommet de la hiérarchie hospitalière. Les médecins seniors qui se font évincer du cursus hospitalier ont officiellement le doit de s'installer comme médecin spécialiste en secteur libéral. En pratique, ils ne le font jamais à cause de l'opposition de leurs confrères. Les médecins généralistes libéraux et les consultants ne leur envoient pas de patients car ils les jugent incompétents. Enfin en Grande-Bretagne comme ailleurs, les médecins généralistes sont les médecins qui ont bénéficié de la formation hospitalière la plus courte. Ils comprennent en leur rang à la fois les personnes qui n'ont pas voulu se spécialiser, et celles qui ont été évincées de la carrière hospitalière. Dominique Broclain (1994) a montré que dans ce pays comme en France, les médecins généralistes se plaignent de l'existence d'une hiérarchie de prestige en faveur des médecins spécialistes.

Dans les trois pays, la manière dont les médecins juniors et seniors sont évalués dans le service influence leur futur mode d'exercice (hospitalier ou non), la nature de leur future activité (spécialisée ou pas) et leur statut social. Dans les trois pays, les médecins hospitalo-universitaires peuvent sélectionner leurs futurs successeurs parce qu'ils forment tous les médecins de leur pays, médecins salariés et libéraux, spécialistes et généralistes, et parce que les médecins généralistes de ces trois pays sont des médecins qui n'ont pas pu ou pas voulu se spécialiser.. Ce dernier point a été analysé par Isabelle Baszanger $(1979,1981)$, qui a montré que les médecins généralistes français ont une "socialisation ratée" dans l'institution hospitalo-universitaire. Ils sont issus d'un système de formation qui n'est pas fait pour eux, et qui fonde sa légitimité sur le prestige intellectuel associé à la logique scientifique. Ce mode de socialisation marginalise les généralistes et scelle la supériorité de la médecine hospitalière sur l'ensemble de la profession.

\section{Pourquoi les différences transnationales dans les relations de travail ?}

Dans les groupes de médecins étudiés, la qualité des relations de travail est liée aux enjeux de carrière. Les perspectives professionnelles pour les médecins juniors dépendent de deux éléments : la politique des chefs de service en matière d'orientation de l'activité médicale et l'environnement administratif et sanitaire de l'hôpital.

\section{Priorité ou non à la recherche}

Comme Antoinette Chauvenet (1973) dans les hôpitaux universitaires français des années 1970, nous avons observé deux types de chefs de service, les uns se spécialisant dans l'étude de cas de pathologies complexes, les autres pas. Pour la période contemporaine, Annick Valette (1994b) a constaté cette même dichotomie parmi les médecins d'hôpitaux généraux français. Ceux-ci présentent un comportement identique à celui de leur confrères hospitalo-universitaires. Notre enquête montre que cette distinction, qu'Antoinette Chauvenet appelle une différence d'idéologie 
médicale, existe dans les trois pays, et qu'elle a des répercussions sur la nature des relations dans tout le groupe médical concerné.

Dans les services de Francfort et Paris, les chefs de service ne développent pas d'activité de recherche et traitent les cas neurologiques les plus fréquents. C'est le type de service le plus conflictuel et le plus difficile à vivre pour les médecins juniors, voire les médecins seniors, à court et à long terme. Accomplir peu ou pas de travaux de recherche réduit drastiquement leurs perspectives de carrière hospitalière. Ces deux services forment essentiellement de futurs médecins libéraux.

Dans les autres services, tout ou partie de la haute hiérarchie médicale donne une orientation scientifique à son activité. Le sommet de la hiérarchie se spécialise dans le traitement des cas les plus complexes de la discipline. Il est influent dans la communauté des neurologues sur le plan national, voire international, et ouvre des possibilités de carrière hospitalo-universitaires aux médecins seniors et parfois aux médecins juniors. Les relations entre le sommet de la hiérarchie médicale et les médecins seniors et juniors sont harmonieuses dans le type 1 , où cette politique de spécialisation se déploie sans entrave, et ne sont bonnes qu'en partie dans le type 3 , où l'environnement oppose des obstacles à cette politique.

\section{Imposer ses choix à son environnement}

La capacité de la haute hiérarchie médicale à imposer ses choix à son environnement distingue le type 1 et 2 du type 3 . Dans les services de type 1 et 2 , rien ne s'oppose à la politique du sommet de la hiérarchie médicale en matière d'orientation de l'activité du service. La haute hiérarchie médicale peut donc développer une activité scientifique dans le type 1, ou ne pas en faire dans le type 2, mettant en œuvre des politiques de recrutement de patients différentes. Une des seules contraintes que subissent ces deux types de services réside, selon le témoignage des médecins, dans l'insuffisance des structures d'accueil des patients en aval, qui oblige parfois les médecins à augmenter la durée de séjour des patients, mais c'est une contrainte minime qui ne modifie pas fondamentalement la politique d'admission.

En revanche dans les services de type 3, l'environnement contrarie la volonté de la haute hiérarchie médicale d'orienter l'activité vers la recherche. Dans le cas de Munich, les médecins sont confrontés à la concurrence d'autres établissements qui hospitalisent les patients, et à l'opposition des neurologues libéraux, des caisses d'assurance-maladie et de la direction de l'hôpital, quant au développement des consultations externes. Dans le cas de Londres, l'administration locale de la santé contrôle de manière étroite le recrutement des patients de l'hôpital par le biais d'une commission interne à l'établissement. Les neurologues sont obligés d'accueillir des patients du service des urgences, mêmes si ceux-ci ne relèvent pas de la neurologie.

Dans l'ensemble, et bien qu'il ne faille pas en tirer de conclusions hâtives, la haute hiérarchie médicale des six services est relativement libre d'imposer ses choix à son environnement. Parmi les groupes médicaux étudiés, seuls deux (un en Allemagne et un en Grande-Bretagne) se heurtent à un environnement hostile à leur orientation. Dans ces deux cas, l'environnement gêne le développement de la politique choisie par la haute hiérarchie médicale, mais ne l'interdit pas. Le sommet de la hiérarchie médicale bénéficie d'une autonomie certaine dans l'orientation qu'il donne à son activité. Personne ne peut l'obliger à faire de la recherche, ou inversement à ne pas en faire. Certains médecins extérieurs au service peuvent exercer un contrôle indirect sur l'orientation de l'activité, par exemple en n'y adressant pas leurs patients, en le rendant accessible aux patients des Urgences ou en influençant à la baisse le tarif de remboursement des consultations externes, mais la 
portée de ce contrôle est limitée. Les acteurs extérieurs aux services étudiés ne semblent pas exercer de contrôle effectif sur le travail clinique et l'activité de recherche. Mais ceci demanderait à être confirmé dans une étude plus large, analysant les rapports entre l'hôpital, l'université, l'administration de tutelle et les organismes payeurs dans plusieurs sites 8 .

\section{Conclusion :}

Les relations de travail dans les groupes de médecins présentent des caractéristiques semblables dans les six services étudiés en Allemagne, France et Grande-Bretagne. L'absence de particularités nationales dans les modes de coopération est d'autant plus frappante que nous en avons relevées au cours de la même enquête dans le groupe des infirmières, et dans les relations entre les infirmières et les médecins. De plus certains auteurs ont montré des spécificités dans la représentation politique de la profession médicale au niveau national et dans le rôle qu'elle joue dans les systèmes de santé (Jobert et Steffen, 1994 ; Hassenteufel, 1994). Ceci pose des problèmes théoriques de décalage entre les visions microsociales et macrosociales de la profession médicale (Vassy, 1999).

Nous avons montré que l'origine des similitudes dans les relations de travail au quotidien réside dans l'existence de règles qui régissent de manière identique les carrières médicales dans les trois pays. L'accès aux postes hospitalo-universitaires est déterminé par la haute hiérarchie médicale des services de soins, qui sélectionne les médecins juniors et seniors. Le recrutement est le plus souvent endogène, la hiérarchie recrutant de préférence des candidats qu'elle a déjà eu l'occasion de voir travailler dans son service. Les candidats malchanceux se tournent principalement vers la médecine libérale, qu'elle soit spécialisée ou généraliste. On retrouve là des phénomènes déjà décrits en France, comme la sélection par l'échec des médecins généralistes (Baszanger 1979) et l'hospitalocentrisme (Arliaud, 1987).

Dans notre échantillon, la qualité des relations de travail varie en fonction de la politique de la haute hiérarchie médicale. Celle-ci choisit de participer ou non à la compétition internationale pour la production de connaissances neurologiques spécialisées. Quand elle ne le fait pas, elle bloque les perspectives de carrière hospitalo-universitaire pour les médecins juniors et seniors. Les deux services qui présentent ces caractéristiques sont les plus conflictuels de notre échantillon. Dans deux autres services, la haute hiérarchie médicale a choisi d'entreprendre une activité de recherche mais elle se heurte à l'opposition d'une partie de son environnement, ce qui réduit les possibilités de spécialisation pour les médecins juniors et génère des tensions.

Cependant dans la plupart des cas, le sommet de la hiérarchie médicale bénéficie d'une grande liberté dans l'orientation de l'activité de son service. L'autonomie, que Freidson a décrit comme l'une des principales caractéristiques de la profession médicale (Freidson, 1984), est particulièrement claire dans le domaine du recrutement et de la sélection des médecins hospitalouniversitaires.

\section{Bibliographie :}

- Michel Arliaud (1987) Les médecins, Paris, La Découverte.

\footnotetext{
${ }^{8}$ Des pistes de recherche ont déjà été ouvertes sur la situation française. Voir par exemple F. Engel, J.Cl. Moisdon, et D. Tonneau (1992), Annick Valette (1994a et 1994b) et André-Pierre Contandriopoulos, Yves Souteyrand (dir) (1996)
} 
- Isabelle Baszanger (1979) Des généralistes en particulier : une approche biographique des processus de socialisation professionnelle Thèse de doctorat de sociologie, sous la direction de Cl. Herzlich, Paris, EHESS

- Isabelle Baszanger (1981) "Socialisation professionnelle et contrôle social, le cas des étudiants en médecine futurs généralistes" Revue Française de Sociologie XXII p.223-245

- Marianne Binst (1990) Du mandarin au manager hospitalier, Paris, L'Harmattan

- Marianne Binst, François-Xavier Schweyer (1995) La Santé, otage de son système, Paris, Interéditions

- Dominique Broclain (1994) "La médecine générale en crise ?" in Pierre Aïach et Didier Fassin Les métiers de la santé, Enjeux de pouvoir et quête de légitimité Paris, Economica p. 121-160

- Theodore Caplow, Reece J. Mc Gee (1968) "Publish or Perish" in Barney G. Glaser (dir) Organizational Careers. A Sourcebook for Theory Chicago, Aldine p.227-229.

- Antoinette Chauvenet (1973) "Idéologies et statuts professionnels chez les médecins hospitaliers", Revue française de sociologie XIV, p. 61-76

- André-Pierre Contandriopoulos, Yves Souteyrand (dir) (1996) L'hôpital stratège, Dynamiques locales et offre de soins Paris, John Libbey

- Frédéric Engel, J.Cl. Moisdon, et D. Tonneau (1992) Contrainte affichée ou contrainte réelle ? Analyse de la régulation du système hospitalier français Cahier de recherche $n^{\circ} 1$, Centre de Gestion Scientifique de l'Ecole des Mines de Paris.

- Eliot Freidson (1984) La profession médicale, Paris, Payot

- Barney G. Glaser (dir) (1968) Organizational Careers. A Sourcebook for Theory Chicago, Aldine Publishing Co.

- William A. Glaser (1963) "American and Foreign Hospitals, Some Sociological comparisons" in Eliot Freidson The Hospital in Modern Society New York, The Free Press p.37-72

- Oswald Hall (1949) "Types of medical careers" American Journal of Sociology November vol LV n³ p.243-253

- Oswald Hall (1970) "Les étapes d'une carrière médicale" in Cl. Herzlich (1970) Médecine, maladie et société op. cit. p.209-223 Première parution (1948) "The stages of a medical career" American Journal of Sociology vol 53, p.327-336

- Patrick Hassenteufel (1994) La profession médicale face à l'Etat, une comparaison France-Allemagne.

Institutionnalisation de la représentation et politique de la santé, Thèse de doctorat en science politique sous la direction de M. P. Birnbaum, Université de Paris I

- Bruno Jobert et Monika Steffen (dir) (1994) "Les politiques de santé en France et en Allemagne" Espace Social Européen $n^{\circ} 258$, dossier spécial nº

- Annick Valette (1994a) "La formation de l'offre de soins à l'hôpital public" op. cit.

- Annick Valette (1994b) Formations des trajectoires d'offre de soins : les interactions hôpital-environnement, Thèse de doctorat de gestion sous la direction de G. de Pouvourville, Université Paris-Dauphine.

- Carine Vassy (1997) Le travail en équipe à l'hôpital. Comparaison de l'organisation de six services de neurologie en Allemagne, France et Grande-Bretagne. Thèse de sociologie sous la direction de E. Friedberg, Institut d'Etudes Politiques de Paris

- Carine Vassy (1999) « Travailler à l'hôpital en Europe. Apport des comparaisons internationales à la sociologie des organisations. » Revue française de sociologie, XL, 2, p.325-356 